\title{
Uso de preservativos por alunos de cursos de saúde em uma universidade pública
}

\section{Use of condoms by students in health courses at a public university}

\author{
Renato da Costa Teixeira ${ }^{1}$, Ellen do Socorro Cruz de Maria ${ }^{2}$, Fernanda Jardim da Silva ${ }^{3}$, Kátia \\ Simone Kietzer ${ }^{4}$, Érica Feio Carneiro Nunes ${ }^{5}$, Fabiana do Socorro da Silva Dias Andrade ${ }^{6}$, José \\ Wagner Cavalcante Muniz ${ }^{7}$
}

\section{Resumo}

\begin{abstract}
As infecções pelo Vírus da Imunodeficiência Humana atingem índices alarmantes no mundo, principalmente em jovens, uma vez que o uso de preservativo é menor entre os jovens. O objetivo desse estudo foi avaliar a principal fonte de orientação sobre o uso de preservativos entre estudantes dos cursos de saúde da Universidade do Estado do Pará, sua frequência de uso e as razões do não uso. Trata-se de um estudo transversal, prospectivo e descritivo que incluiu estudantes matriculados em cursos de graduação em saúde usando um questionário on-line. Participam do estudo 86 alunos. O tema sexualidade foi considerado fechado na família. A universidade foi apontada como a principal fonte de informação referente ao tema. A maioria afirmou ter utilizado o preservativo na última relação e sempre se previne, porém confessou já ter negligenciado o uso em algum momento por descuido, confiança no parceiro ou não querer usar.. Conclui-se que os participantes fazem uso do método de forma irregular, mesmo referindo terem elevado conhecimento sobre a questão. Apesar de serem futuros multiplicadores de informações, os alunos pesquisados demonstraram não fazer na prática aquilo que irão ensinar a seus futuros pacientes.
\end{abstract}

Palavras chave: Ensino superior. Sexualidade. Educação em saúde.

\begin{abstract}
Human immunodeficiency virus infections are reaching alarming rates worldwide, especially among young people, as the use of condoms is lower among young people. The aim of this study was to evaluate the main source of guidance on the use of condoms among students of health courses at Pará State University, frequency of use, and reasons for not using them. This was a cross-sectional, prospective, descriptive study that included students enrolled in undergraduate health courses using an online questionnaire. Eighty-six students participated by answering the questionnaire. The subject of sexuality was considered an unmentioned topic in the family. The university was considered the main source of information on this topic. Most claimed to have used a condom the last time they had sexual intercourse and to be always preventive; however they already admitted to have neglected the use, on occasion, only because they had confidence in their partnet, or simply because they did not want to use one. In conclusion, participants used the method irregularly, despite indicating strong knowledge on the matter. Although they are future information disseminators, were inconsistent in practicing what they will one day be teaching to their future patients.
\end{abstract}

Keywords: Higher education. Sexuality. Health education.

\footnotetext{
${ }^{1}$ Doutorado em Educação pela Pontifícia Universidade Católica do Rio de Janeiro, Rio de Janeiro, Brasil. Professor adjunto do Centro de Ciências Biológicas e da Saúde da Universidade do Estado do Pará, Belém, Pará, Brasil. E-mail: renatocteixeira@uepa.br

${ }^{2}$ Acadêmica de Fisioterapia pela Universidade do Estado do Pará, Belém, Pará, Brasil.

${ }^{3}$ Graduada em Ciências Naturais - Habilitação em Biologia, pela Universidade do Estado do Pará, Belém, Pará, Brasil.

${ }^{4}$ Doutorado em Neurociências e Biologia Celular pela Universidade Federal do Pará, Belém, Pará, Brasil. Professora adjunta do Centro de Ciências Biológicas e da Saúde da Universidade Federal do Pará, Belém, Pará, Brasil.

${ }_{5}^{5}$ Doutoranda em Ciências da Reabilitação pela Universidade Nove de Julho, São Paulo, Brasil. Docente da Universidade do Estado do Pará, Belém, Pará, Brasil.

${ }^{6}$ Doutora em Ciências pela Universidade Federal de São Paulo, São Paulo, Brasil. Fisioterapeuta do município de São Francisco de Paula, Rio Grande do Sul, Brasil.

${ }^{7}$ Doutorado em Neurociências e Biologia Celular pela Universidade Federal do Pará, Belém, Pará, Brasil. Professor Adjunto da Universidade do Estado do Pará, Belém, Pará, Brasil.
} 


\section{Introdução}

As infecções sexualmente transmissíveis (IST's) são um conjunto de infecções distintas que têm em comum o fato de serem transmitidas através do contato sexual, podendo atingir qualquer pessoa independente da sua classe social, sexo, raça, nível de escolaridade, entre outras características sociais, são um dos problemas de saúde pública mais comuns em todo o mundo ${ }^{(1)}$ e dentre elas encontra-se a infecção pelo Vírus da Imunodeficiência Humana (HIV-AIDS).

A minimização de danos e a prevenção da infecção por HIV-AIDS são estratégias primordiais para enfrentar a epidemia, principalmente em função do aumento da prevalência da infecção na população jovem. ${ }^{(2-3)}$ Dados do Ministério da Saúde revelam que em oito anos foram registrados mais de 30 mil casos da doença nesse grupo da população. Se em 2004 havia 9,6 casos de AIDS em cada grupo de 100 mil habitantes de 15 a 24 anos, em 2013 o índice saltou para 12,7 segundo o Ministério da Saúde. ${ }^{(2)}$ Ao todo, 4.414 jovens foram detectados com o vírus em 2013, enquanto em 2004 haviam sido 3.453.

Dados do Ministério da Saúde ${ }^{(4)}$ apontam resultados relevantes para avaliar a situação das IST's no país. A prevalência de IST bacteriana foi de $14,4 \%$, e a das virais, $41,9 \%$. Por outro lado, a prevalência da infecção pelo HPV é elevada e afeta fundamentalmente os adolescentes e jovens, sugerindo que a infecção produz-se em geral em idade mais precoce, no início das relações sexuais. As maiores taxas de infecção gonocócica e por clamídia foram observadas nas pessoas mais jovens.

Além dessas, outra IST que preocupa os gestores de saúde publica é a infecção pelo HIV-AIDS. Segundo o boletim epidemiológico HIV-AIDS de 2014, desde o início da epidemia no Brasil até junho de 2014, foram registrados 757.042 casos. ${ }^{(5)}$

Os jovens são considerados um grupo de grande risco epidemiológico devido à variabilidade de parceiros sexuais, o uso de drogas injetáveis, a baixa idade das primeiras relações sexuais e por não receberem a orientação sexual correta na escola e em casa, onde, muitas vezes o assunto ainda é considerado um tabu. (6) A sexualidade está presente em toda a trajetória de vida do ser humano, mas a sua afirmação acontece na adolescência. No entanto, o desenvolvimento desta nem sempre é acompanhado de um amadurecimento afetivo e cognitivo, o que torna esta uma etapa de extrema vulnerabilidade a riscos como gravidez indesejada e as IST's como a infecção por HIV-AIDS.

No Brasil, o preservativo foi e continua sendo a principal aposta no campo da prevenção das IST's. A idade de início da atividade sexual tem diminuído ao longo dos anos e a chance do uso de preservativo é menor quanto mais baixa for a idade e a escolaridade.

(7) Tal assertiva nos leva a inferir que quanto maior for a escolaridade maior a chance de uso do preservativo e, desta forma, alunos de nível superior como os de graduação, teriam como prática frequente o uso de preservativos em suas relações sexuais e este fato poderia estar relacionado ao aprendizado acadêmico.

Deve-se considerar também que os universitários dos cursos de saúde, serão os futuros profissionais que além de cuidar da saúde da população, serão formadores de opinião, responsáveis por multiplicar ações educativas e, assim, podemos afirmar que carregam grandes responsabilidades para com a sociedade.

Assim, este estudo teve como objetivo avaliar qual a principal fonte de orientação sobre o uso de preservativos por universitários dos cursos de saúde de uma universidade pública, sua frequência de uso assim como as razões para uso e não do mesmo.

\section{Material e Método}

O presente estudo foi aprovado pelo Comitê de Ética em Pesquisa do Campus II da Universidade do Estado do Pará sob o Parecer $n^{\circ} 1.223 .733$ de 11 /09/2015 (CAAE no 48339015.6.0000.5174).

Foi um estudo transversal, prospectivo e descritivo onde foram incluídos alunos regulamente matriculados nos cursos de graduação em Medicina, Enfermagem, Fisioterapia, Terapia Ocupacional, Educação Física e Biomedicina, pertencentes ao Centro de Ciências Biológicas e da Saúde, da Universidade do Estado do Pará, na cidade de Belém do Pará, que aceitaram participar voluntariamente da pesquisa. Tendo como critério de exclusão, alunos sexualmente inativos.

A pesquisa se deu por meio do preenchimento de um questionário on-line através da plataforma Google Forms, que não informa ao pesquisador os dados de identificação do respondente, garantindo-se assim a confidencialidade desse tipo de informação necessária ao tipo de estudo. Os pesquisadores foram às turmas explicar o teor da pesquisa, e em seguida, com o consentimento dos alunos, foram enviados os formulários para os e-mails coletivos de cada turma. Antes de responder ao questionário o participante 
deveria ler o TCLE e concordar em participar. Caso não concordasse o questionário não era aberto e a pesquisa terminava. O formulário ficou disponível para respostas durante o período de seis meses (setembro de 2015 a março de 2016) e era composto por 14 questões, sendo 4 referentes ao perfil do participante e 10 relacionadas ao comportamento sexual e ao conhecimento auto referido sobre IST's. A análise das respostas foi feita de maneira descritiva, e os dados tabelados em uma planilha disponibilizada pelo Google Forms.

\section{Resultados}

Acessaram o questionário 87 estudantes, porém, um não consentiu a pesquisa, sendo o número amostral, portanto, de 86 estudantes.

Houve predomínio do sexo feminino $(60,6 \%)$ sobre o sexo masculino $(38,3 \%), 1,1 \%$ não declarou o sexo. A média de idade, em anos, foi de: $20,20 \pm$ 2,24. A maior participação foi de acadêmicos do curso de Medicina, seguido por Fisioterapia, Enfermagem, Educação Física, Terapia Ocupacional e Biomedicina, conforme exposto no Gráfico 1.

Gráfico 1 - Distribuição percentual dos participantes que responderam ao questionário por curso.

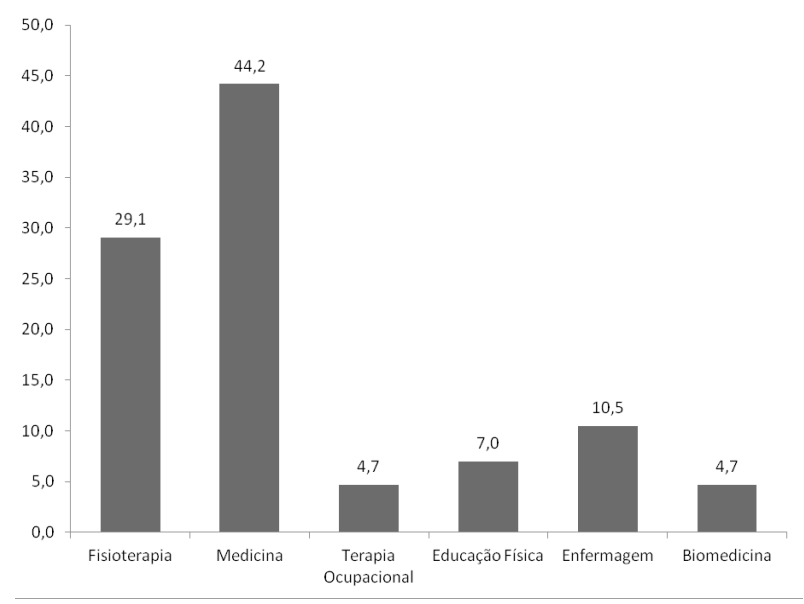

Quanto ao semestre letivo, a distribuição dos participantes foi a seguinte: $1^{\circ}$ semestre $(13,9 \%), 2^{\circ}$ semestre $(16,3 \%), 3^{\circ}$ semestre $(12,8 \%), 4^{\circ}$ semestre $(22,1 \%), 5^{\circ}$ semestre $(3,5 \%), 6^{\circ}$ semestre $(10,5 \%), 7^{\circ}$ semestre $(12,8 \%), 8^{\circ}$ semestre $(4,6 \%), 10^{\circ}$ semestre $(2,3 \%)$ e não declarado $(1,1 \%)$.

Em relação à abertura do ambiente familiar para o tema sexualidade, muito fechado $(10,5 \%)$, fechado
$(31,4 \%)$, nem fechado nem aberto $(30,2 \%)$, aberto $(17,4 \%)$ e muito aberto (10,5\%). A universidade foi apontada como a principal fonte de informação sobre IST's, seguida da internet, os dados de todas as alternativas estão dispostos no Gráfico 2.

Gráfico 2 - Distribuição percentual da principal fonte de informação sobre IST's, segundo os participantes.

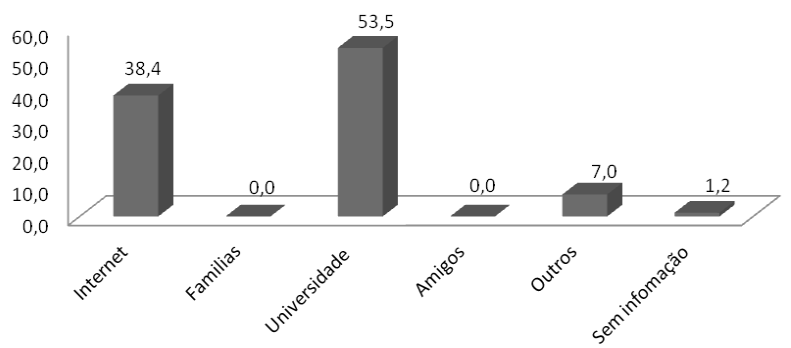

Ainda sobre a aquisição de informações, $77,9 \%$ já participaram de algum evento sobre educação sexual e $22,1 \%$ nunca participaram. Ao serem questionados sobre seus conhecimentos acerca das IST's, um maior número de respondentes considera ter um nível alto ou médio como demonstra a Tabela 1.

Tabela 1 - Nível de conhecimento sobre IST's autorreferido pelos participantes

\begin{tabular}{lll}
\hline Variável resposta & $\mathbf{N}$ & $\mathbf{\%}$ \\
\hline Muito baixo & 0 & 0 \\
Baixo & 1 & 1,2 \\
Médio & 28 & 32,6 \\
Alto & 50 & 58,1 \\
Muito alto & 7 & 8,1 \\
\hline Total & $\mathbf{8 6}$ & $\mathbf{1 0 0}$ \\
\hline
\end{tabular}

Sobre a influência do uso de preservativos em seus relacionamentos $77,9 \%$ afirmaram que o fato de confiar no parceiro não dispensa a prevenção e o mesmo número, $77,9 \%$, não desconfiaria da fidelidade deste, caso houvesse insistência para o uso. Em contrapartida, $22,1 \%$ afirmaram dispensar o preservativo quando existe confiança e também $22,1 \%$ desconfiariam em 
caso de insistência. A maioria afirmou ter utilizado o preservativo na última relação $(74,4 \%)$ e $45,3 \%$ sempre se previne. A Tabela 2 expõe a frequência do uso de preservativo relatada pelos participantes.

Tabela 2 - Frequência de uso de preservativos apontada pelos participantes

\begin{tabular}{lll}
\hline Variável resposta & $\mathbf{N}$ & $\mathbf{\%}$ \\
\hline Sem informação & 6 & 7,0 \\
Quase nunca & 12 & 14,0 \\
Na maior parte das vezes & 29 & 33,7 \\
Sempre & 39 & 45,3 \\
\hline Total & $\mathbf{8 6}$ & $\mathbf{1 0 0}$ \\
\hline
\end{tabular}

Dentre os entrevistados, $56(65,07 \%)$ confessaram já ter deixado de utilizar em algum momento o preservativo. Os motivos estão apresentados na tabela 3.

Tabela 3 - Motivos para o não uso do preservativo, segundo os participantes.

\begin{tabular}{lll}
\hline Variável resposta & $\mathbf{N}$ & $\mathbf{\%}$ \\
\hline $\begin{array}{l}\text { Eu não tinha no momento / Esquecimento } \\
\text { Não quero usar / Uso de outro método }\end{array}$ & 17 & 30,4 \\
$\begin{array}{l}\text { contraceptivo / Experimentar sem / } \\
\text { Insistência do parceiro / Consenso do casal }\end{array}$ & 15 & 26,8 \\
Confiança no parceiro & 11 & 19,6 \\
Melhor prazer & 4 & 7,1 \\
Desconforto / Dor & 3 & 5,4 \\
Perda de prazer & 2 & 3,6 \\
Eu não sei como responder & 1 & 1,8 \\
Pressa & 1 & 1,8 \\
Alergia & 1 & 1,8 \\
Eu não gosto & 1 & 1,8 \\
\hline Total & $\mathbf{5 6}$ & $\mathbf{1 0 0}$ \\
\hline
\end{tabular}

\section{Discussão}

Conhecer o comportamento sexual de acadêmicos dos cursos da saúde torna-se importante, pois estes são futuros profissionais responsáveis por conscientizar e fornecer esclarecimento à população sobre questões relacionadas à saúde, dentre estas a utilização correta dos métodos preventivos, devendo, portanto, ser bem informados e livres de preconceitos. $^{(8)}$

As respostas dadas pelos universitários revelam uso inconsistente dos preservativos; 45,3\% afirmaram usar "sempre", no entanto um percentual maior $(47,6 \%)$ afirmou usar "a maior parte das vezes" ou "quase nunca" mostrando que ainda deixam de utilizar em determinado momento. Bezerra et al.(9) em pesquisa com universitários observaram tal inconsistência na prevenção, assim como, uma atitude levemente desfavorável ao uso do preservativo entre os sexualmente ativos.

Em um estudo sobre a frequência do uso de preservativos por jovens universitários, ${ }^{(1)}$ foi evidenciado que, apesar de todos os informantes terem demonstrado conhecimento quanto à importância do uso rotineiro do preservativo, a minoria disse aderir ao uso em todas as relações sexuais. Dentre os motivos mais recorrentes estão: não possuir o preservativo no momento da relação e ter confiança no parceiro, resultado esse igual ao encontrado em nosso estudo. Outro motivo que deve ser considerado é a perda de sensibilidade, este é recorrente na literatura como no estudo de Silva ${ }^{(10)}$ no qual quase $70 \%$ consideram que o preservativo diminui o prazer.

Quando se fala na aquisição de informações o fato da universidade ser apontada como maior fonte, faz refletir sobre o papel que o ambiente educacional pode ter na discussão acerca do conhecimento sobre as IST's. Pois estes são meios privilegiados para planejar medidas que estimulem o autocuidado e que proporcionem a participação dos alunos na construção da sua própria saúde.(11) Por meio de suas funções de ensino, pesquisa, assistência e extensão, as Instituições de Ensino Superior (IES) possuem participação fundamental nas estratégias de prevenção. ${ }^{(12)}$

$\mathrm{O}$ fato de um número importante ter declarado possuir alto nível de conhecimento sobre as IST'S, o que também foi encontrado por Silva, ${ }^{(12)}$ foi um aspecto positivo observado pelo estudo, que pode ser considerado como um avanço conquistado pelas várias campanhas de conscientização. Porém, as variáveis conhecimento e uso de preservativos não são diretamente proporcionais. ${ }^{(6)}$ Nesse sentido, a relação existente entre a vulnerabilidade dos jovens, principalmente acadêmicos de cursos ligados à área da saúde, com o sentimento de conhecimento sobre o assunto, favorecendo práticas duvidosas e um comportamento sexual perigoso. ${ }^{(10)}$ Podemos afirmar desse modo, que um elevado nível de conhecimentos 
não determina individualmente comportamentos sem riscos. Por esse motivo, é importante que haja a preocupação, não somente sobre a quantidade da informação transmitida, mas sobre a forma como ela é transmitida. ${ }^{(13)}$ Pois, sabe-se que a prevenção não se dá apenas com o conhecimento das formas de contágio, mas, também, com a habilidade para colocar em prática medidas e atitudes favoráveis à sua adoção. ${ }^{(12)}$

Quando questionados sobre a relação do recurso com relacionamentos amorosos, a maioria afirmou que a confiança não dispensa a prevenção, um ponto relevante que contraria o senso comum onde se tem que a solicitação para uso do preservativo pode despertar desconfiança quanto à fidelidade dos parceiros. ${ }^{(1)}$

Além de tudo que foi exposto, a pouca abertura do ambiente familiar para conversas sobre sexualidade ainda parece ser um problema recorrente, o que sugere que os pais podem não estar se comunicando de maneira eficaz com seus filhos, e possuem dificuldades para conversar sobre os aspectos necessários para uma prática sexual segura. ${ }^{(14)}$

Esta falta de diálogo pode ser um dos fatores responsáveis pelo não uso da contracepção, o que é resultado de uma cultura onde sexo é um assunto ainda envolto por preconceitos. Com isso, jovens não encontram espaço para falar sobre suas dúvidas e acabam por receber e transmitir informações distorcidas, pelo fato de os seus responsáveis acabarem ignorando e reprimindo-os quanto aos seus posicionamentos sobre a questão. ${ }^{(6,15)}$ Por isso é de extrema importância o investimento em ações educativas sobre sexualidade e ISTS dentro da universidade, com a abordagem de temas relacionadas principalmente ao uso do preservativo. ${ }^{(8)}$

A universidade é uma grande fonte para produção e busca de conhecimento, mas os investimentos nessa produção devem sempre aumentar, principalmente quanto a temas relacionados à sexualidade como as ITS's e o uso de preservativos, uma vez que esses temas ainda geram dúvidas, desconfortos e incertezas, o que leva a doenças crônicas e mesmo a morte.

Medidas estratégicas de enfrentamento do problema devem ser incentivadas, mas não somente nas universidades, devem também alcançar todos os estudantes, para que desde cedo cresçam com a firmeza do cuidado próprio e do outro.

\section{Conclusão}

Investigar o uso ou não de preservativo em jovens é necessário para que políticas públicas possam ser melhor estruturadas. Em nosso estudo encontramos que a principal fonte de orientação sobre o uso de preservativo pelos participantes é a universidade e que a amostra em questão, apesar de referir ter elevado conhecimento sobre as IST's, faz uso de forma irregular do preservativo por não ter no momento da relação, por confiança no parceiro ou por opção. Parece ainda haver uma dificuldade no diálogo com os familiares sobre questões relacionadas à sexualidade, o que pode estar contribuindo para uma prática sexual insegura. Desta forma, espera-se contribuir para que medidas de promoção e educação em saúde possam ser difundidas nos cursos de saúde, visto que estes acadêmicos serão multiplicadores de conhecimento, devendo começar por si mesmo.

\section{Agradecimentos}

Agradecemos a Universidade do Estado do Pará pelo financiamento parcial do estudo através de bolsa de assistência estudantil aos alunos envolvidos na coleta de dados.

\section{Referências}

1 Santos NA, Rebouças LCC, Boery RNO, Boery EG, Silva SS. Adesão de universitários ao uso dos preservativos. Rev. Saúde Comunitária. 2009;5(2):116-127.

2 Ministério da Saúde (BR). Recomendações para a atenção integral a adolescentes e jovens vivendo com HIV/Aids. Brasília (DF): Ministério da Saúde; 2013.

3 Pinheiro TF, Calazans GJ, Ayres JRCM. Uso de camisinha no Brasil: um olhar sobre a produção acadêmica acerca da prevenção de HIV/Aids (2007-2011). Temas Psicol. 2013;21(3):815-836.

4 Ministério da Saúde (BR). Prevalências e frequências relativas de doenças sexualmente transmissíveis (IST) em populações selecionadas de seis capitais brasileiras, 2005. Brasília (DF): Ministério da Saúde; 2008. 
5 Ministério da Saúde (BR). Boletim epidemiológico: HIV-AIDS. Brasília (DF): Ministério da Saúde, 2014.

6 Jardim VMJ, Nominato LT, Ghetti PAO, Lauriano MM, Gadêlha TA, Schimitt PM, et al. O conhecimento e o uso de preservativo por adolescentes: estudo comparativo em uma escola particular e pública. Rev. Cient. Fac. Med. Campos. $2013 ; 8(1)$.

7 Taquete ST. Epidemia de HIV/Aids em adolescentes no Brasil e na França: semelhanças e diferenças. R. Saude Soc. 2013 mar;22(2):618-628. Doi: 10.1590/ S0104-12902013000200029.

8 Jaques AE, Valera IMA, Zaramello WMA, Dantas FS, Pãoeagua EC, Gerbasi ARV. Opinião de acadêmicos de enfermagem sobre o uso de preservativos. Arq. Ciênc. Saúde UNIPAR. 2014;18(2):95-100.

9 Bezerra EO, Chaves ACP, Pereira MLD, Melo FRG. Análise da vulnerabilidade sexual de estudantes universitários ao hiv/aids. Rev. Rene. 2012;13(50):1121-31.

10 Silva VOFL. Conhecimento de estudantes universitários sobre a transmissão das IST/VIHSIDA e o uso do preservativo. [Dissertação]. Cabo Verde: Universidade de Cabo Verde; 2014.

11 Reis M, Ramiro L, Matos LG, Diniz JÁ, Simões C. Relação entre atitudes sexuais, conhecimentos e atitudes sobre VIH/Sida na orientação sexual. Psic. Saúde \& Doenças. 2013;14(1):141-151

12 Silva AL. Conhecimento, diagnóstico sorológico e uso de preservativo: instrumentos de prevenção da aids entre os estudantes universitários. [Dissertação]. Vila Real: Universidade de Trás-osMontes e Alto Douro; 2014.

13 Martins AT, Nunes C, Muñoz-Silva A, SánchezGarcía M. Fontes de informação, conhecimentos e uso do preservativo em estudantes universitários do Algarve e de Huelva. Psicol. 2008;39(1).
14 Moser AG, Reggiani C, Urbanetz A. Comportamento sexual de risco entre estudantes universitárias dos cursos de ciências da saúde. Rev. Assoc. Med. Bras. 2007 abr;53(2):116-121. Doi:10.1590/S0104-42302007000200014.

15 Albuquerque GA, Belém JM, Nunes JFC, Alves MJH, Feitosa FRA, Queiroz CMHT, et al. Saberes e práticas sexuais de adolescentes do sexo masculino: impactos na saúde. RECOM. 2014;4(2):1146-1160. 